\title{
SETS OF DETERMINATION FOR HARMONIC FUNCTIONS
}

\author{
STEPHEN J. GARDINER
}

\begin{abstract}
Let $h$ denote a positive harmonic function on the open unit ball $B$ of Euclidean space $\mathbf{R}^{n} \quad(n \geq 2)$. This paper characterizes those subsets $E$ of $B$ for which $\sup _{E} H / h=\sup _{B} H / h$ or $\inf _{E} H / h=\inf _{B} H / h$ for all harmonic functions $H$ belonging to a specified class. In this regard we consider the classes of positive harmonic functions, differences of positive harmonic functions, and harmonic functions with a one-sided quasi-boundedness condition. We also consider the closely related question of representing functions on the sphere $\partial B$ as sums of Poisson kernels corresponding to points in $E$.
\end{abstract}

\section{INTRODUCTION AND MAIN RESULTS}

Let $B(X, r)$ denote the open ball in $\mathbf{R}^{n}$ with center $X$ and positive radius $r$, and define $B=B(O, 1)$, where $O$ denotes the origin of $\mathbf{R}^{n}$. The Poisson kernel for $B$ with pole $Y$ in $\partial B$ is given by $P(Y, X)=\sigma_{n}^{-1}\left(1-|X|^{2}\right)|X-Y|^{-n}$, where $\sigma_{n}$ is the surface area of $\partial B$. For any $h$ in the class $\mathscr{H}^{+}$of all positive harmonic functions on $B$, let $\mu_{h}$ denote the corresponding measure on $\partial B$ such that $h(X)=\int_{\partial B} P(Y, X) d \mu_{h}(Y)$.

Let $h \in \mathscr{H}^{+}$. The main purpose of this paper is to establish necessary and sufficient conditions on a subset $E$ of $B$ for the equality $\sup _{E} H / h=\sup _{B} H / h$ (or $\inf _{E} H / h=\inf _{B} H / h$ ) to hold for all harmonic functions $H$ belonging to certain classes. It will be shown that what is required is that there is "enough of $E$ " near every, or almost every $\left(\mu_{h}\right), Y$ in $\partial B$, depending on the class considered. More precisely, if $E \subseteq B$ and $0<\rho<1$, we define a set $E_{\rho}=$ $\bigcup_{X \in E} B(X, \rho(1-|X|))$, where $|X|$ denotes the Euclidean norm of $X$, and a function

$$
E_{\rho}^{*}(Y)=\int_{E_{\rho}}|X-Y|^{-n} d X \quad(Y \in \partial B),
$$

which takes values in $[0, \infty]$. By "enough of $E$ " near $Y$ in $\partial B$ we mean $E_{\rho}^{*}(Y)=\infty$ for some $\rho$. The significance of $E_{\rho}^{*}(Y)$ is shown by the following result.

Theorem A. Let $E \subseteq B$ and $Y \in \partial B$. The following are equivalent:

$$
\begin{gathered}
\inf _{X \in E} \frac{H(X)}{P(Y, X)}=\inf _{X \in B} \frac{H(X)}{P(Y, X)} \quad \text { for all } H \text { in } \mathscr{H}^{+} ; \\
E_{\rho}^{*}(Y)=\infty \quad \text { for some } \rho \text { in }(0,1) \\
E_{\rho}^{*}(Y)=\infty \quad \text { for all } \rho \text { in }(0,1) .
\end{gathered}
$$

Received by the editors October 3, 1990 and, in revised form, April 5, 1991. 1991 Mathematics Subject Classification. Primary 31B05. 
Theorem A is essentially due to Beurling [2] when $n=2$ and was established for higher dimensions by Dahlberg [6]. An alternative proof has been given by Sjögren [13]. A further equivalent condition, involving "separated sequences" of points in $E$, can also be found in the cited papers. We remark that, of course, the value of the infimum in (i) above is $\mu_{H}(\{Y\})$.

It is an interesting fact that the characterization of sets $E$ which determine $\sup _{B} H / h$ is closely related to the representation of functions on the sphere $\partial B$ in terms of Poisson kernels corresponding to points in $E$. Thus we also consider representations of positive continuous functions $f$ on $\partial B$, and of functions $f$ in $L^{1}\left(\mu_{h}\right)$, of the form

$$
f=\sum_{k} \lambda_{k} P\left(\cdot, X_{k}\right)
$$

where $\left\{X_{k}\right\} \subseteq E$.

Let $\mathscr{S} \mathscr{H}$ denote the class of all subharmonic functions $s$ on $B$ such that $s^{+}$has a harmonic majorant on $B$, and let $\mathscr{H}$ denote the subclass of harmonic members of $\mathscr{S} \mathscr{H}$. Thus $\mathscr{H}$ consists of the harmonic functions on $B$ which can be written as $H_{1}-H_{2}$, where $H_{1}, H_{2} \in \mathscr{H}^{+}$. Also, let $\mathscr{A}(f ; X, r)$ denote the mean (when defined) of a function $f$ over the ball $B(X, r)$.

Theorem 1. Let $E \subseteq B$. The following are equivalent:

(iv) for every positive continuous function $f$ on $\partial B$ there exist sequences $\left(\lambda_{k}\right)$ in $(0,+\infty)$ and $\left(X_{k}\right)$ in $E$ such that

$$
f(Y)=\sum_{k=1}^{\infty} \lambda_{k} P\left(Y, X_{k}\right) \quad(Y \in \partial B) .
$$

Theorem 1 (equivalence of (i), (iii), and (iv)) was recently established when $n=2$ by Hayman and Lyons [12, Theorems 2, 3]. Bonsall and Walsh [5, Theorem 10], also working in the plane, established the equivalence of (i) and (iv) using a different approach, and asked [5, p. 432] if this could be extended to give a short proof of the Hayman-Lyons result. The proof of Theorem 1 (given in §3) answers their question affirmatively, as well as extending the contents of [12] to higher dimensions. The extra condition (ii) contains the statement " $\sup _{E} H / h=\sup _{B} H / h$ for all $H$ in $\mathscr{H}$ and all $h$ in $\mathscr{H}^{+}$," because of the mean value property of harmonic functions. We note that Essén [9] $(n=2)$ and Dudley-Ward [8] ( $n \geq 2$ and more general domains) given proofs of the equivalence of (i), (iii), and (iv) independently of our work.

Let $\Phi$ denote the class of all convex strictly increasing functions $\phi:[0, \infty) \rightarrow$ $[0, \infty)$ such that $\phi(x) / x \rightarrow \infty$ as $x \rightarrow \infty$. (Doob [7] describes these as uniform integrability test functions. An example is the function $\phi(x)=x^{p}$ when $p>1$.) 
Further, if $h \in \mathscr{H}^{+}$, let $\mathscr{S} \mathscr{H}_{h, \Phi}$ denote the class of all subharmonic functions $s$ on $B$ such that $h \phi\left(s^{+} / h\right)$ has a harmonic majorant on $B$ for some $\phi$ in $\Phi$. (We recall [10] that the convex increasing property of $\phi$ ensures that $h \phi\left(s^{+} / h\right)$ is itself subharmonic on $B$.) As before, $\mathscr{H}_{h, \Phi}$ denotes the class comprising the harmonic members of $\mathscr{S} \mathscr{H}_{h, \Phi}$. In particular, $\mathscr{H}_{h, \Phi}$ contains all harmonic functions $H$ on $B$ such that $H / h$ is bounded above.

Theorem 2. Let $E \subseteq B$ and let $h$ be a positive harmonic function on $B$ with corresponding measure $\mu_{h}$. The following are equivalent:

$$
\begin{aligned}
& \inf _{X \in E} \frac{H(X)}{h(X)}=\inf _{X \in B} \frac{H(X)}{h(X)} \quad \text { for all } H \text { in } \mathscr{H}^{+} ; \\
& \sup _{X \in E} \frac{H(X)}{h(X)}=\sup _{X \in B} \frac{H(X)}{h(X)} \quad \text { for all } H \text { in } \mathscr{H}_{h, \Phi} ; \\
& \quad \sup _{X \in E} \frac{\mathscr{A}(s ; X, \kappa(1-|X|))}{h(X)}=\sup _{X \in B} \frac{s(X)}{h(X)} \\
& \text { for all } s \text { in } \mathscr{S}_{h} \mathscr{H}_{h} \text { and all } \kappa \text { in }(0,1) ; \\
& \text { there exists } \rho \text { in }(0,1) \text { such that } E_{\rho}^{*}(Y)=\infty
\end{aligned}
$$

We note that Theorem 2 (equivalence of (i) and (iv)) is a generalization of Theorem A. It is not hard to see that the value of the infimum in (i) is equal to the essential infimum of the Radon-Nikodým derivative of the absolutely continuous component of $\mu_{H}$ relative to $\mu_{h}$. (See [7, 1.XII.19].)

Corollary 1. Let $E \subseteq B$ and let $h$ be a positive harmonic function on $B$ with corresponding measure $\mu_{h}$. If there exists $\rho$ in $(0,1)$ such that $E_{\rho}^{*}(Y)=\infty$ for almost every $\left(\mu_{h}\right) \quad Y$ in $\partial B$, then for every $f$ in $L^{1}\left(\mu_{h}\right)$ there exist $\left(\lambda_{k}\right)$ in $l_{1}(\mathbf{C})$ and a sequence $\left(X_{k}\right)$ of points in $E$ such that

$$
f=\sum_{k=1}^{\infty} \lambda_{k} P\left(\cdot, X_{k}\right) / h\left(X_{k}\right)
$$

(convergence in the sense of $\left.L^{1}\left(\mu_{h}\right)\right)$. Further,

$$
\|f\|_{L^{\prime}\left(\mu_{h}\right)}=\inf \left\{\sum\left|\lambda_{k}\right|: \text { (1) holds for some sequence }\left(X_{k}\right) \text { in } E\right\} .
$$

The converse of Corollary 1 is false in general, as can easily be seen by considering the case $h=P(Z, \cdot)$ for any $Z$ in $\partial B$. However, more can be said when $h \equiv 1$. Let $\sigma$ denote surface area measure on $\partial B$. A positive harmonic function on $B$ is called quasi-bounded if it is the limit of an increasing sequence of bounded positive harmonic functions.

Corollary 2. Let $E \subseteq B$. The following are equivalent:

$$
\inf _{X \in E} H(X)=\inf _{X \in B} H(X) \quad \text { for all } H \text { in } \mathscr{H}^{+} \text {; }
$$

(ii) $\sup _{X \in E} H(X)=\sup _{X \in B} H(X)$ for all harmonic functions $H$ which have a quasi-bounded harmonic majorant on $B$;

(iii) $\sup _{X \in E} H(X)=\sup _{X \in B} H(X)$ for all bounded positive harmonic functions $H$ on $B$; 
(iv) there exists $\rho$ in $(0,1)$ such that $E_{\rho}^{*}(Y)=\infty$ for almost every ( $\sigma) Y$ in $\partial B$;

(v) for almost every $(\sigma) Y$ in $\partial B$ there is a sequence of points in $E$ which converges to $Y$ nontangentially;

(vi) for every $f$ in $L^{1}(\sigma)$ there exist $\left(\lambda_{k}\right)$ in $l_{1}(\mathbf{C})$ and a sequence $\left(X_{k}\right)$ of points in $E$ such that

$$
f=\sum_{k=1}^{\infty} \lambda_{k} P\left(\cdot, X_{k}\right)
$$

and, further,

$$
\|f\|_{L^{1}(\sigma)}=\inf \left\{\sum\left|\lambda_{k}\right|:(2) \text { holds for some sequence }\left(X_{k}\right) \text { in } E\right\} .
$$

Corollary 2 extends a recent result of Bonsall [4], who showed in the case of the unit disc that (v) and (vi) are both equivalent to saying that $\sup _{E}|H|=$ $\sup _{B}|H|$ for all bounded harmonic functions $H$. The equivalence of (iv) and (v) when $n=2$ is due to Walsh and Hayman (see [11]).

Finally we add below a further equivalent condition to Theorem A.

Theorem 3. Let $E \subseteq B$ and $Y \in \partial B$. Then $E_{\rho}^{*}(Y)=\infty$ for some $\rho$ in $(0,1)$ if and only if

$$
\liminf _{\substack{X \rightarrow Y \\ X \in E}} \frac{H(X)}{1-|X|}=\liminf _{\substack{X \rightarrow Y \\ X \in B}} \frac{H(X)}{1-|X|} \quad \text { for all } H \text { in } \mathscr{H}^{+} .
$$

Theorems 1-3 are proved in $\S \S 3-5$, and the two corollaries are deduced in $\S 6$.

\section{Preliminary MATerial}

In this section we record several facts that will be used in the proofs of the main results. The first is simply a version of Harnack's inequality: if $H \in \mathscr{H}^{+}$ and $\rho \in(0,1)$, then

$$
\frac{1-\rho^{2}}{(1+\rho)^{n}} \leq \frac{H(X)}{H(Z)} \leq \frac{1-\rho^{2}}{(1-\rho)^{n}} \quad \text { when } X \in B(Z, \rho(1-|Z|)) .
$$

The second is an estimate used by Beardon [1, Lemma 3.2] to prove an analogue of Harnack's inequality applicable to volume means of positive superharmonic functions. We give a proof here for the sake of completeness.

Lemma 1. Let $u$ be a positive superharmonic function on $B(Z, R)$, let $r \in(0, R)$ and $\delta \in(0,1)$. If $X \in B(Z, \min \{R-r, \delta r\})$, then $u(X) \geq$ $(1-\delta)^{n} \mathscr{A}(u ; Z, r)$.

In fact,

$$
\begin{aligned}
u(X) & \geq \mathscr{A}(u ; X, r) \geq n \sigma_{n}^{-1} r^{-n} \int_{B(Z,(1-\delta) r)} u(X) d X \\
& =(1-\delta)^{n} \mathscr{A}(u ; Z,(1-\delta) r) \geq(1-\delta)^{n} \mathscr{A}(u ; Z, r)
\end{aligned}
$$


The next result is due to Dahlberg [6, Theorem 2] when $n \geq 3$. A closely related result had previously been established for $n=2$ by Beurling [2, Lemma I]. An alternative proof, valid when $n \geq 2$, can be found in Sjögren [13, Theorem 2]. We express it here in terms of the minimal thinness. (An account of the minimal fine topology can be found in Doob [7, I.XII].)

Theorem B. Let $Y \in \partial B$. If $E$ is a measurable subset of $B$ such that $\int_{E}|X-Y|^{-n} d X=\infty$, then $E$ is not minimally thin at $Y$.

Our final main tool, which is true more generally than stated below, can be found in Doob [7, 1.XII.17, Application].

Theorem $\mathrm{C}$. Let $h$ be a positive harmonic function on $B$ with corresponding measure $\mu_{h}$, and let $E$ be a subset of $B$ such that almost every $\left(\mu_{h}\right) \quad Y$ in $\partial B$ is a minimal fine limit point of $E$. If $u$ is a positive superharmonic function on $B$ such that $u \geq C h$ on $E$, where $C>0$, then $u \geq C h$ on $B$.

\section{Proof of Theorem 1}

3.1. We begin by showing that (iii) implies (ii). Suppose that (iii) holds, that $s \in \mathscr{S} \mathscr{H}$ and that $\kappa, \delta \in(0,1)$. We can write $s$ as $H-u$, where $H \in \mathscr{H}^{+}$ and $u$ is a positive superharmonic function on $B$. By Theorem A, $E_{\rho}^{*}(Y)=\infty$ for all $Y$ in $\partial B$, where $\rho=\min \{1-\kappa, \delta \kappa\}$. If $X \in B(Z, \rho(1-|Z|))$, then Lemma 1 applied to $u$ on $B(Z, 1-|Z|)$ shows that

$$
\mathscr{A}(u ; Z, \kappa(1-|Z|)) \leq(1-\delta)^{-n} u(X) \text {. }
$$

Let $h \in \mathscr{H}^{+}$. We define

$$
c=\sup _{Z \in E} \frac{\mathscr{A}(s ; Z, \kappa(1-|Z|))}{h(Z)} \quad \text { and } \quad d=\sup _{Z \in B} \frac{s(Z)}{h(Z)} .
$$

If $d<\infty$, then $s \leq d h$ in $B$, so

$$
\mathscr{A}(s ; Z, \kappa(1-|Z|)) \leq d \mathscr{A}(h ; Z, \kappa(1-|Z|))=d h(Z) \quad(Z \in B),
$$

and hence $c \leq d$. Thus, if $c=\infty$, we must have $d=\infty$ also.

Now suppose that $0 \leq c<\infty$. Clearly

$$
H(Z) \leq \operatorname{ch}(Z)+\mathscr{A}(u ; Z, \kappa(1-|Z|)) \quad(Z \in E) .
$$

Applying (3) to $h$ and $H$, and using (4) and (5), we have

$$
\frac{(1-\rho)^{n}}{1-\rho^{2}} H(X) \leq c \frac{(1+\rho)^{n}}{1-\rho^{2}} h(X)+(1-\delta)^{-n} u(X) \quad\left(X \in E_{\rho}\right) .
$$

From Theorem B, every $Y$ in $\partial B$ is a minimal fine limit point of $E_{\rho}$. Thus, by Theorem $\mathrm{C}$, (6) holds for all $X$ in $B$. Letting $\delta$ tend to 0 (so that $\rho \rightarrow 0$ also), we deduce that $s=H-u \leq c h$ on $B$. Thus $d \leq c$. From the previous paragraph we now have $d=c$.

If $c<0$, an analogous argument can be applied to the inequality

$$
H(Z)-\operatorname{ch}(Z) \leq \mathscr{A}(u ; Z, \kappa(1-|Z|)) \quad(Z \in E)
$$

in place of (5). We have now established (ii).

3.2. The mean value property of harmonic functions shows that (ii) (with $h \equiv 1$ ) implies (i). 
3.3. An argument of Bonsall and Walsh [5, Theorem 10 (ii) $\Rightarrow(\mathrm{i})]$ (originally stated for $n=2$, but valid also in the present context) establishes that (i) implies (iv).

3.4. It remains to show that (iv) implies (iii). We fix $Z$ in $\partial B, H$ in $\mathscr{H}^{+}$, and define $c=\inf _{E} H / P(Z, \cdot)$. Now fix $X$ in $B$. By (iv) there exist sequences $\left(\lambda_{k}\right)$ in $(0, \infty)$ and $\left(X_{k}\right)$ in $E$ such that

$$
P(Y, X)=\sum_{k=1}^{\infty} \lambda_{k} P\left(Y, X_{k}\right) \quad(Y \in \partial B) .
$$

Integrating with respect to $d \mu_{H}(Y)$ we have, by monotone convergence,

$$
H(X)=\sum_{k=1}^{\infty} \lambda_{k} H\left(X_{k}\right) \geq c \sum_{k=1}^{\infty} \lambda_{k} P\left(Z, X_{k}\right)=c P(Z, X) .
$$

Since $X$ was an arbitrary point of $B$, we now have $\inf _{B} H / P(Z, \cdot)=c$. Since $H$ was an arbitrary member of $\mathscr{H}^{+}$, it follows from Theorem A that $E_{\rho}^{*}(Z)=\infty$ for all $\rho$ in $(0,1)$. Since $Z$ was an arbitrary point of $\partial B$, (iii) is proved.

\section{Proof of Theorem 2}

4.1. Let $S \in \mathscr{S}^{\prime}{ }_{h, \Phi}$. Then there exists $\phi$ in $\Phi$ and $H_{0}$ in $\mathscr{H}^{+}$such that $h \phi\left(s^{+} / h\right) \leq H_{0}$ on $B$. Hence $s^{+}$is majorized on $B$ by $h \phi^{-1}\left(H_{0} / h\right)$, which is a superharmonic function (cf. the argument in [10]). Let $H$ be the least harmonic majorant of $s^{+}$on $B$. Then $s^{+} \leq H \leq h \phi^{-1}\left(H_{0} / h\right)$, whence $h \phi(H / h) \leq H_{0}$ on $B$. It follows (see [7, 1.IX.12]) that $\mu_{H}$ is absolutely continuous with respect to $\mu_{h}$ on $\partial B$. Thus we can write $s=H-u$, where $u$ is a positive superharmonic function on $B$ and $H$ is as above.

With this extra ingredient, the proof that (iv) implies (iii) follows the pattern of $\S 3.1$. (Almost every $\left(\mu_{H}\right) \quad Y$ in $\partial B$ is a minimal fine limit point of $E_{\rho}$ since almost every $\left(\mu_{h}\right) \quad Y$ in $\partial B$ is.)

4.2. It follows from the mean value property of harmonic functions that (iii) implies (ii).

4.3. If $H \in \mathscr{H}^{+}$, then $-H \in \mathscr{H}_{h, \Phi}$, so (ii) implies (i).

4.4. It remains to prove that (i) implies (iv). Suppose that (iv) does not hold. If $E$ is empty, then (i) fails since $\inf _{E} H / h=\infty$. Thus we can assume that $E \neq \varnothing$. If $\bar{E} \subseteq B$, let $H$ be any member of $\mathscr{H}^{+}$which is not a multiple of $h$, and put $c=\inf _{E} H / h$. Applying the minimum principle to $H-c h$, it is clear that (i) fails. In what follows we can thus assume that $E$ is not compactly contained in $B$. Further, it is enough to show that (i) fails when $E$ is replaced by $E \backslash B(O, R)$ for some $R$ less than 1 .

Let $\varepsilon$ be a fixed positive number and define

$$
f_{k}(Y)=\int_{E_{\rho} \backslash B(O, 1-1 / k)}|X-Y|^{-n} d X \quad(Y \in \partial B)
$$

when $k=2,3, \ldots$. Since $\left(f_{k}\right)$ decreases to the zero function on a set of positive $\mu_{h}$-measure, we can choose $k_{\varepsilon}$ large enough so that the set $D=\{Y \in$ $\left.\partial B: f_{k_{\varepsilon}}(Y)<\varepsilon\right\}$ has positive $\mu_{h}$-measure. Thus, replacing $E$ by $E \backslash B(O, R)$ 
for a suitably chosen $R$ in $(0,1)$, we can assume that $E_{\rho}^{*}(Y)<\varepsilon$ for all $Y$ in a set $D$ of positive $\mu_{h}$-measure. Further, by choosing $\varepsilon$ to be sufficiently small we can ensure that $\Gamma_{Y} \cap E_{\rho}=\varnothing$ for all $Y$ in $D$, where

$$
\Gamma_{Y}=\{X: 1-|X|>|X-Y| / 2\} \text {. }
$$

We now define a measure $\lambda$ on the Borel subsets $A$ of $\partial B$ by putting $\lambda(A)$ equal to the $n$-dimensional Lebesgue measure of $\left\{X \in E_{\rho}: X /|X| \in A\right\}$. For each $Y$ in $D$ let

$$
H_{Y}(X)=\int_{\partial B}|Y-Z|^{-n} P(Z, X) d \lambda(Z) \quad(X \in B) .
$$

Since $\Gamma_{Y} \cap E_{\rho}=\varnothing$ we have

$$
\int_{\partial B}|Y-Z|^{-n} d \lambda(Z) \leq C(n) E_{\rho}^{*}(Y)<C(n) \varepsilon<\infty,
$$

(we use $C(n)$ to denote a positive constant depending on $n$, not necessarily the same on any two occurrences) and so $H_{Y}$ is harmonic on $B$ for each $Y$ in $D$. Also, if $X \in E$, then

$$
\begin{aligned}
H_{Y}(X) & \geq C(n)(1-|X|)^{1-n}|X-Y|^{-n} \int_{B(X, \rho(1-|X|))} d Z \\
& \geq C(n) \rho^{n} P(Y, X) .
\end{aligned}
$$

We now define

$$
H_{1}(X)=\int_{D} P(Y, X) d \mu_{h}(Y), \quad H_{2}(X)=\int_{\partial B \backslash D} P(Y, X) d \mu_{h}(Y),
$$

and

$$
H_{3}(X)=\int_{D} H_{Y}(X) d \mu_{h}(Y)=\int_{\partial B} f(Z) P(Z, X) d \lambda(Z),
$$

where

$$
f(Z)=\int_{D}|Y-Z|^{-n} d \mu_{h}(Y) \quad(Z \in \partial B) .
$$

(The change in order of integration in (9) is valid because the integrand is positive.) Clearly $H_{1}$ and $H_{2}$ are harmonic functions on $B$. Since

$$
\begin{aligned}
\int_{\partial B} f(Z) d \lambda(Z) & =\int_{D}\left\{\int_{\partial B}|Y-Z|^{-n} d \lambda(Z)\right\} d \mu_{h}(Y) \\
& \leq C(n) \varepsilon \mu_{h}(D)<\infty,
\end{aligned}
$$

by (7), so too is $H_{3}$. We define $H=H_{2}+H_{3}$. If $X \in E$, then (8) shows that $H_{3}(X) \geq C(n, \rho) H_{1}(X)$, and so

$$
\frac{H(X)}{h(X)} \geq \frac{H_{2}(X)+C(n, \rho) H_{1}(X)}{h(X)} \geq C(n, \rho) \quad(X \in E),
$$

since $h=H_{1}+H_{2}$.

The proof will be complete if we can show that $\inf _{B} H / h=0$. To do this, we apply the Fatou-Naïm-Doob boundary limit theorem [7, 1.XII.19] to observe that

$$
\operatorname{mf} \lim _{X \rightarrow Y} \frac{H_{2}(X)}{H_{1}(X)}=0 \quad \text { for a.e. }\left(\mu_{h}\right) Y \text { in } D
$$


so that

$$
\operatorname{mf} \lim _{X \rightarrow Y} \frac{h(X)}{H_{1}(X)}=1 \text { for a.e. }\left(\mu_{h}\right) Y \text { in } D,
$$

where the prefix "mf" signifies that the attached limit is with respect to the minimal fine topology on $\bar{B}$. Also, the function $f$ defined in (10) is valued $\infty$ at almost every $\left(\mu_{h}\right) Z$ in $D$. (To see this, apply [7, 1.V.11] to the set $D \times\{(0,0)\}$, which is polar in $\mathbf{R}^{n+2}$.) Thus, from (11), the measure $\lambda$ is singular with respect to the restriction of $\mu_{h}$ to $D$, and so

$$
\operatorname{mf} \lim _{X \rightarrow Y} \frac{H_{3}(X)}{H_{1}(X)}=0 \text { for a.e. }\left(\mu_{h}\right) Y \text { in } D \text {. }
$$

Combining (12), (13), (14), we have

$$
\mathrm{mf} \lim _{X \rightarrow Y} \frac{H(X)}{h(X)}=\mathrm{mf} \lim _{X \rightarrow Y}\left(\frac{H_{2} / H_{1}+H_{3} / H_{1}}{h / H_{1}}\right)(X)=0
$$

for a.e. $\left(\mu_{h}\right) Y$ in $D$.

Since $\mu_{h}(D)>0$, it follows that $\inf _{B} H / h=0$, as required. The proof of Theorem 2 is now complete.

\section{Proof of Theorem 3}

5.1. The only if part of Theorem 3 will be proved first. Suppose that $E_{\rho}^{*}(Y)=$ $\infty$ for some $\rho$ in $(0,1)$. By Theorem $A, E_{\rho}^{*}(Y)=\infty$ for all $\rho$ in $(0,1)$. Let $H \in \mathscr{H}^{+}$and define $F(X)=H(X) /(1-|X|)$. From $(3), \quad F(X) \geq$ $(1-\rho)(1+\rho)^{-n} F(Z)$ for $X$ in $B(Z, \rho(1-|Z|))$ and so

$$
\liminf _{\substack{X \rightarrow Y \\ X \in E_{\rho}}} F(X) \geq \frac{1-\rho}{(1+\rho)^{n}} \liminf _{\substack{X \rightarrow Y \\ X \in E}} F(X) .
$$

Since (by Theorem B) $E_{\rho}$ is not minimally thin at $Y$, we have

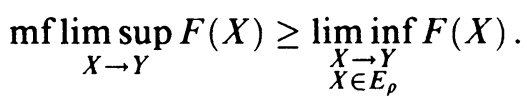

Let $G(\cdot, \cdot)$ denote the Green function for $B$. For any positive superharmonic function $u$ on $B$ it is known (see [7, 1.XII.14]) that $u / G(O, \cdot)$ has minimal fine limit at $Y$ equal to its lower limit there. Since $G(O, X) /(1-|X|)$ has a positive limit as $X \rightarrow Y$, it follows from (15) and (16) that

$$
\liminf _{\substack{X \rightarrow Y \\ X \in B}} F(X) \geq \frac{1-\rho}{(1+\rho)^{n}} \liminf _{\substack{X \rightarrow Y \\ X \in E}} F(X) \geq \frac{1-\rho}{(1+\rho)^{n}} \liminf _{\substack{X \rightarrow Y \\ X \in B}} F(X) .
$$

The argument is completed by letting $\rho$ tend to 0 .

5.2. To prove the if part of Theorem 3, suppose that $E_{\rho}^{*}(Y)<\infty$ for some $\rho$ in $(0,1)$. If $Y$ is not a limit point of $E$, then we interpret the lower limit of any function as $X \rightarrow Y$ along $E$ as $\infty$. In this case, if we take $Z$ in $\partial B \backslash\{Y\}$ and define $H=P(Z, \cdot)$, we have

$$
\liminf _{\substack{X \rightarrow Y \\ X \in B}} \frac{H(X)}{1-|X|}=\left(2 / \sigma_{n}\right)|Y-Z|^{-n}<\liminf _{\substack{X \rightarrow Y \\ X \in E}} \frac{H(X)}{1-|X|} .
$$


We now assume that $Y$ is a limit point of $E$. Since $E_{\rho}^{*}(Y)<\infty$, there is no sequence of points in $E$ which converges nontangentially to $Y$. It follows that $Y$ is not a limit point of $\Gamma_{Y} \cap E_{\rho}$. Thus we can assume, without loss of generality, that $\Gamma_{Y} \cap E_{\rho}=\varnothing$.

Since $E_{\rho}^{*}(Y)<\infty$, there exists a decreasing function $f:(0, \infty) \rightarrow(0, \infty)$ such that $f(t) \rightarrow \infty$ as $t \rightarrow 0+$ and

$$
\int_{E_{\rho}}|X-Y|^{-n} f(|X-Y|) d X<\infty .
$$

Now let $\lambda$ be the measure defined on the Borel subsets $A$ of $\partial B$ by

$$
\lambda(A)=\int_{\left\{X \in E_{\rho}: X /|X| \in A\right\}} f(|X-Y|) d X,
$$

and define $H(X)=\int P(Z, X) d \lambda(Z)$. Since $\Gamma_{Y} \cap E_{\rho}=\varnothing$, it follows from inequality (17) that $\int|Z-Y|^{-n} d \lambda(Z)<\infty$. Hence, by dominated convergence, $H(X) /(1-|X|)$, has a finite radial limit at $Y$.

It is now sufficient to show that $H(X) /(1-|X|) \rightarrow \infty$ as $X$ approaches $Y$ along $E$. In fact, if $X \in E$, then

$$
\begin{aligned}
H(X) & \geq C(n)(1-|X|)^{1-n} \int_{B(X, \rho(1-|X|))} f(|Z-Y|) d Z \\
& \geq C(n) \rho^{n}(1-|X|) f((1+\rho / 2)|X-Y|) .
\end{aligned}
$$

Hence, as $X$ approaches $Y$ along $E$,

$$
\frac{H(X)}{1-|X|} \geq C(n) \rho^{n} f((1+\rho / 2)|X-Y|) \rightarrow \infty,
$$

as required.

\section{Proof of Corollaries 1 and 2}

6.1. The proof of Corollary 1 is based on the following result of Bonsall [3, Theorem 1], and the application given in [3, Corollary 2].

Theorem D. Let $\left(f_{k}\right)$ be a sequence in a Banach space $\mathscr{X}$ such that $\left\|f_{k}\right\| \leq C_{1}$ for all $k$ in $\mathbf{N}$ and $\sup _{k}\left|\psi\left(f_{k}\right)\right| \geq C_{2}\|\psi\|$ for all $\psi$ in $\mathscr{X}^{*}$, where $C_{1}, C_{2}>0$. Then every $f$ in $\mathscr{X}$ is of the form $f=\sum \lambda_{k} f_{k}$ for some $\left(\lambda_{k}\right)$ in $l_{1}(\mathbf{C})$, and also

$$
C_{2} \inf \left\{\sum\left|\lambda_{k}\right|\right\} \leq\|f\| \leq C_{1} \inf \left\{\sum\left|\lambda_{k}\right|\right\},
$$

where the infima are taken over all possible choices of $\left(\lambda_{k}\right)$ in the representation of $f$.

Now let $E$ and $h$ be as in the statement of Corollary 1, let $\left\{X_{k}\right\}$ be a countable dense subset of $E$, and put $f_{k}(Y)=P\left(Y, X_{k}\right) / h\left(X_{k}\right)$ for $Y$ in $\partial B$. Clearly each $f_{k}$ has norm 1 in $L^{1}\left(\mu_{h}\right)$. Next, let $\psi \in\left(L^{1}\left(\mu_{h}\right)\right)^{*}$. By the Riesz representation theorem there exists $g$ in $L^{\infty}\left(\mu_{h}\right)$ such that

$$
\psi(f)=\int_{\partial B} f(Y) g(Y) d \mu_{h}(Y) \quad\left(f \in L^{1}\left(\mu_{h}\right)\right)
$$


and $\|\psi\|=\|g\|_{L^{\infty}\left(\mu_{h}\right)}$. Using first Theorem $2(($ iv $) \Rightarrow($ ii $))$ and then the fact [7, 1.XII.19] that

$$
\operatorname{mf} \lim _{X \rightarrow Y} \frac{\psi(P(\cdot, X))}{h(X)}=g(Y) \quad \text { a.e. }\left(\mu_{h}\right) Y \text { in } \partial B,
$$

we obtain

$$
\sup _{k \in \mathbf{N}}\left|\psi\left(f_{k}\right)\right|=\sup _{X \in B} \frac{|\psi(P(\cdot, X))|}{h(X)}=\|g\|_{L^{\infty}\left(\mu_{h}\right)}=\|\psi\| .
$$

Hence Theorem D can be applied, with $C_{1}=1=C_{2}$, to obtain Corollary 1 .

6.2. Corollary 2 will now be proved. If $H$ is a harmonic function which has a quasi-bounded harmonic majorant on $B$, then [7, 1.IX.12] there exists $\phi$ in $\Phi$ such that $\phi\left(H^{+}\right)$has a harmonic majorant on $B$. With this observation, the equivalence of (i), (ii), and (iv) is immediate from Theorem 2 (with $h \equiv 1$, so that $\left.\mu_{h}=\sigma\right)$. Further, it is easy to see that (v) implies (iv), and Corollary 1 shows that (iv) implies (vi). It remains to check that (vi) implies (iii) and that (iii) implies (v).

So suppose that (vi) holds, let $H$ be a bounded positive harmonic function on $B$, and put $c=\sup _{E} H$. Also, let $\varepsilon>0$. If we fix $X$ in $B$, then there exist $\left(\lambda_{k}\right)$ in $l_{1}(\mathbf{R})$ and a sequence $\left(X_{k}\right)$ of points in $E$ such that

$$
P(\cdot, X)=\sum_{k=1}^{\infty} \lambda_{k} P\left(\cdot, X_{k}\right) \quad \text { and } \quad \sum_{k=1}^{\infty}\left|\lambda_{k}\right|<1+\varepsilon .
$$

Integrating with respect to $d \mu_{h}(Y)$ and using the monotone convergence theorem, we have

$$
H(X) \leq \sum_{k=1}^{\infty} \lambda_{k}^{+} H\left(X_{k}\right) \leq c \sum_{k=1}^{\infty} \lambda_{k}^{+}<c(1+\varepsilon) .
$$

Since $X$ and $\varepsilon$ were arbitrary, we now have $\sup _{B} H=c$, which proves (iii).

Finally, we prove that (iii) implies (v) using an argument similar to that in Bonsall [4, Theorem 3]. Suppose that (v) fails. When $Y \in \partial B$ and $0<a<1$, let $\Gamma_{Y}^{a}$ denote the region $\{X: a>1-|X|>|X-Y| / 2\}$. By choosing $a$ to be sufficiently small we can select a subset $D$ of $\partial B$, of positive (but not full) surface area measure, such that $\Gamma_{Y}^{a} \cap E=\varnothing$ for all $Y$ in $D$. We define

$$
H[A](X)=\int_{A} P(Y, X) d \sigma(Y) \quad(X \in B)
$$

for any Borel subset $A$ of $\partial B$, and

$$
d=\inf \{H[\partial B \cap B(X, 2(1-|X|))](X): 1-a<|X|<1\} .
$$

Clearly $d>0$. Hence $H[D]$ is a bounded positive harmonic function on $B$ such that $\sup _{B} H[D]=1$ and

$$
\sup _{E} H[D] \leq \max \left\{1-d, \sup _{E \cap \bar{B}(O, 1-a)} H[D]\right\}<1,
$$

contradicting (iii). This completes the proof of Corollary 2.

Added in proof. Professor Maz'ya has informed me that his paper, Beurling's theorem on a minimum principle for positive harmonic functions (in Russian), Zap. Nauch. Sem. LOMI 30 (1972), 76-90 (English transl. in J. Soviet Math. 4 (1976), 367-379) extended Beurling's minimum principle to higher dimensions; cf. Theorems A and B above. 


\section{REFERENCES}

1. A. F. Beardon, Montel's theorem for subharmonic functions and solutions of partial differential equations, Proc. Cambridge Philos. Soc. 69 (1971), 123-150.

2. A. Beurling, A minimum principle for positive harmonic functions, Ann. Acad. Sci. Fenn. Ser. AI, no. 372 (1965), 7 pp.

3. F. F. Bonsall, Decompositions of functions as sums of elementary functions, Quart. J. Math. Oxford (2) 37 (1986), 129-136.

4. __ Domination of the supremum of a bounded harmonic function by its supremum over a countable subset, Proc. Edinburgh Math. Soc. 30 (1987), 471-477.

5. F. F. Bonsall and D. Walsh, Vanishing $l^{1}$-sums of the Poisson kernel, and sums with positive coefficients, Proc. Edinburgh Math. Soc. 32 (1989), 431-447.

6. B. Dahlberg, A minimum principle for positive harmonic functions, Proc. London Math. Soc. (3) 33 (1976), 238-250.

7. J. L. Doob, Classical potential theory and its probabilistic counterpart, Springer-Verlag, New York, 1984.

8. N. Dudley-Ward, Doctoral thesis, University of York, 1991.

9. M. Essén, On minimal thinness, reduced functions and Green potentials, Proc. Edinburgh Math. Soc. 36 (1992), 87-106.

10. S. J. Gardiner and M. Klimek, Convexity and subsolutions of partial differential equations, Bull. London Math. Soc. 18 (1986), 41-43.

11. W. K. Hayman, Atomic decompositions, Recent Advances in Fourier Analysis and its Applications (J. S. Byrnes and J. L. Byrnes, eds.), Kluwer, 1990.

12. W. K. Hayman and T. J. Lyons, Bases for positive continuous functions, J. London Math. Soc. 42 (1990), 292-308.

13. P. Sjögren, Une propriété des fonctions harmoniques positives, d'après Dahlberg, Séminaire de Théorie du Potentiel, Lecture Notes in Math., vol. 563, Springer-Verlag, Berlin, 1976, pp. 275-282.

Department of Mathematics, University College, Dublin, Ireland

E-mail address: gardiner@irlearn.ucd.ie 